\title{
Effect of Waste Heat from Egbin Thermal Plant on the Plankton and Macrobenthos of Lagos Lagoon
}

\author{
Nwankwo, Dike Ikegwu (Corresponding author) \\ Department of Marine Sciences, University of Lagos, Nigeria. \\ Tel: 234-703-845-2541 E-mail: dikenwankwo2@yahoo.com
}

Chukwu, Lucian Obinna

Department of Marine Sciences, University of Lagos, Nigeria

Osiegbu, Godson

Department of Marine Sciences, University of Lagos, Nigeria

Igho-Okor, Ejiro

Department of Marine Sciences, University of Lagos, Nigeria

Abiola, Olamide

Department of Marine Sciences, University of Lagos, Nigeria

Received: October 26, 2013 Accepted: November 15, $2013 \quad$ Published: June 15, 2014

doi:10.5296/jee.v5i1.5810 URL: http://dx.doi.org/10.5296/jee.v5i1.5810

\begin{abstract}
The effect of waste heat discharge on the water chemistry, plankton and benthic fauna in the Lagos lagoon at Egbin was investigated for six months (February - July 2000) over three dry month (February - April) and three wet months (May - July).Water temperature were high $\left(27^{\circ} \mathrm{C}-40^{\circ} \mathrm{C}\right)$ and decreased spatially from station A to C. Transparency was higher in the dry months than wet months. The highest transparency $(196 \mathrm{~cm})$ was recorded in May at station A while the lowest $(66 \mathrm{~cm})$ was recorded in June at station B. Similarly, the highest Total
\end{abstract}




\section{Macrothink}

Dissolved Solids $\left(394 \mathrm{mgL}^{-1}\right)$ was recorded in July. The site was acidic $(<6.93)$ all through the sampling period Conductivity $\left(<2.5 \mathrm{~cm}^{-1}\right)$ and salinity $(<12.1 \%$ ) values followed seasonal trends and were higher at station $\mathrm{B}$. The lowest dissolved oxygen value $\left(3.2 \mathrm{mgL}^{-1}\right)$ was recorded in May while higher nutrient values $\left(\mathrm{NO}_{3}-\mathrm{N} 3.98 \mathrm{mg} / \mathrm{L}^{-1}, \mathrm{PO}_{4}-\mathrm{P} 0.64 \mathrm{mg} / \mathrm{L}^{-1}, \mathrm{SO}_{4}\right.$ $1135 \mathrm{mg} / \mathrm{L}^{-1}$ ) were recorded in March at station B. Heavy metals and oil and grease values were higher in the dry months. The biota spectrum comprised 83 phytoplankton species, 23 zooplankton species and five benthic fauna. High cyanobacteria specie, richness was recorded in elevated water temperatures $\left(>27^{\circ} \mathrm{C}\right)$, high transparency and low dissolved oxygen content $\left(3.50 \mathrm{mg} / \mathrm{L}^{-1}\right)$ at stations A and B. Plankton species were more abundant in the wet than dry months. Aulacosera, Spirogyra, Acartia, Paracalanus and Cyclops were prevalent plankton forms while Pachymalania, Tymapanotho and Aloides were frequent benthic taxa especially in the wet months. The high number of empty shells was more discernable in stations $\mathrm{A}$ and $\mathrm{B}$.

Keywords: Plankton, Macrobenthos, Temperature, Dissolved oxygen, Waste heat 


\section{Introduction}

The need to generate power worldwide had given rise to the use of various sources such as nuclear, gas, coal, wind, hydro, thermal and solar. Some of these are not environment friendly and create challenges particularly when the world is facing a major crisis, global warming. According to Thorhaiug (1978), the incidence of waste heat discharge in tropical coastal waters is even more critical as most marine organisms in the region already operate at the upper limits of their temperature tolerance. In the marine environments of estuaries, lagoons and coastal sea water, waste heat discharge may alter the environment in several ways. For instance, the water chemistry may change the status of heavy metals (Swany 1994, Ananthan, et al., 2005) creating favourable loci for opportunistic species and elimination of venerable species. (Abbaspour, 2005). Discharge waste heat may lead to the development of plumes of heated water in discharge zones in estuaries and coastal environments. Stress induced by elevated temperatures could lead to an increase in metabolic rate of marine biota and faster exploitation of food sources. Increased metabolic rate could also shorten the lifespan of primary producers leading to a proliferation of species (Staughan 1980). There is the possibility of anoxic conditions when algal blooms collapse in alteration of species diversity (Surash et al., 1937, Nwankwo and Adesalu, 2010) and collapse of the aquatic environment food network in developing economies with fossil fuel, balancing the energy needs with green environment has been a huge challenges.

In Nigeria, over $85 \%$ of all industries are located in the Lagos area. Some of these industries discharge their waste through drainage systems into nearby storm water drains and coastal waters (Odiete, 1999). Of particular note are wastes from sewage customs, saw mills, breweries and chemical facilities. Also important are gas turbines used for power generation which release waste heat into the aquatic environment. These turbines include adjoining facilities at Ijora in Lagos State, Imo river in River State, Oji river in Enugu State, Ugheli in Delta State and Egbin in Lagos State. The Egbin thermal station with a generation capacity of 1320 watts (Ukuoma, 1989) accounts for a quarter of Nigeria's installed power need. Thermal plants release warmer water as major fallout of the internal combustion process (Ajao et al., 1996, Odiete, 1999). However, the effect of continuous warming up of tropical waters has not received much attention. The aim of this study was to investigate the plankton and benthic fauna diversity at a waste-heat discharge site at Egbin, Lagos.

\section{Materials and Methods}

\subsection{Description of study site}

The coast of South-west Nigeria is characterized by a meandering network, of lagoons and creeks of which the Lagos lagon with a surface area of $208 \mathrm{~km}^{2}$ is the most documented (FAO 1969, Nwankwo, 2004). Egbin, (Fig. I) with coordinates $30^{\circ} 40$ and $6^{0} 34$ is located East of Lagos lagoon. The lagoon is open all year round via the Lagos harbor and experiences semi diurnal tidal regime. Sea water incursion and fresh water from adjourning rivers determine the environment of the area (Nwankwo, 1996). Owing to the dynamics inflow of river and sea water incursion, the Lagos lagoon experiences brackish condition that is more discernable in the dry season (Hill and Webb, 1958; Nwankwo, 1996) and decreases inland. 


\subsection{Collection of Samples}

Duplicate monthly samples were collected for six months (February - July, 2000) between $11.00 \mathrm{~h}$ and $13.00 \mathrm{~h}$. Water samples for some water chemistry determination were collected $20 \mathrm{~cm}$ below the water surface in $500 \mathrm{ml}$ well labeled plastic containers with screw caps and stored away in an ice chest. Plankton samples were collected using a $55 \mu \mathrm{m}$ size standard plankton net tied onto a motorized boat and towed horizontally at low speed ( $<4$ knots) for five minutes. Each haul was concentrated and transferred into a well labeled $500 \mathrm{ml}$ plastic container with screw cap. Each container was preserved in $4 \%$ buffered formalin. Benthic samples were collected using a $0.1 \mathrm{~m}$ Van Veen grab from an anchored boat. On each occasion, three hauls were taken from each station. A haul from each station was washed through a $0.5 \mathrm{~mm}$ mesh sieve in the field. Preservation of biological organisms retained was done in $10 \%$ formalin and stored in well labeled containers. Sediment samples were stored in three identifiable plastic bags while samples for the determination of oil and grease were collected in foils.

\subsection{Physical and Chemical Analyses of Water Samples}

All measurements were made either in the field or on return in the laboratory. Water temperature was measured in situ using a mercury in glass thermometer while transparency was determined using a $20 \mathrm{~cm}$ white and black painted Sechi disc. Total suspended solids and total dissolved solids were determined by filtering a $500 \mathrm{ml}$ sample through a weighed filter paper, evaporating in oven at $105^{\circ} \mathrm{C}$ and reweighing and by evaporating $100 \mathrm{ml}$ filtrate in an oven at $100^{\circ} \mathrm{C}$ respectively. The $\mathrm{pH}$ of water was measured with a Griffin digital $\mathrm{pH}$ meter (Model 80) while dissolved oxygen was measured using a Griffin meter (Model 40). Salinity and conductivity were determined in field using a refractometer and a conductivity meter (HANNA instrument model H18733) respectively. The nitrate-nitrogen and soluble reactives phosphorus were measured by the phenol-disulphonic acid method (Macereth 1971). Sulphate values were estimated using turbidimetric method (APHA, 1998). Heavy metals (Cu, $\mathrm{Fe}, \mathrm{Pb}$ ) were determined using an atomic Absorption Spectrometer (Model 969). Rainfall data were kindly supplied by the Nigerian Institute of Oceanography and Marine Research (NIOMR).

\subsection{Sediment Analysis}

Total organic matter (TOM) was determined using the ash method described in (APHA 1998) while sedimentation texture was determined using a measuring cylinder and the sedimentation techniques as described by Oyenekan (1975).

\subsection{Plankton Analysis}

In the laboratory, each biological sample was concentrated to $20 \mathrm{~cm}$. five drops from a well mixed sample were thoroughly investigated under a wild 22 binocular microscope with a calibrated eye piece. To scan each drop, the microtransect drop count method suggested by Laecky (1936) was used. Relevant text (Hendey 1964, Patrick and Reimer 1966-1975, Van Laningham 1982 for phytoplankton while Olaniyan 1975, Waife and Frid, 2000 were used for Zooplankton identification. 


\subsection{Benthic Analysis}

Preserved benthic organisms were washed in running tap water to remove any preservatives and remaining sediments. The animals were sorted into groups and identified using relevant texts (Edmunds 1978) were used to aid identification. The number of species and individuals per station were counted and recorded as number of individuals/species/station.

\subsection{Community Structure Analysis}

Three community structure indices were used to a assess the community structure. These were Species richness (d) described by Margalef (1951) as d = and Shannon and Weaver (1963) index presented as $\mathrm{Hs}=$ (j) and Equitability

described by Hs

$$
\text { Log2 S }
$$

Where $\mathrm{d}=$ Species richness

$\mathrm{S}=$ Number of species in a population

$\mathrm{N}=$ Total number of individuals in $\mathrm{S}$ species

I = Counts denoting the ith species ranging from $\mathrm{i}-\mathrm{n}$

$\mathrm{Pi}=$ Population that the $\mathrm{ith}$ species representing in terms of numbers of individuals with respect to the total number of individuals in the sampling space as a whole.

Hs $=$ Diversity index

$\mathrm{J} \quad=$ Equitability index

\section{Results}

\subsection{Physico-Chemical Parameters}

Results of the water quality characteristics are presented in Table 1.Water temperatures recorded at the sampling stations were between $27^{\circ} \mathrm{C}$ and $40^{\circ} \mathrm{C}$. The highest water temperature $\left(40^{\circ} \mathrm{C}\right)$ was recorded in May at station $\mathrm{A}$, while the lowest $\left(27^{\circ} \mathrm{C}\right)$ was recorded in February, June and July at station C. Water temperature estimates were highest at station A throughout the study. Transparency values $(66-196 \mathrm{~cm})$ were higher in the dry months than the wet months. The highest value $(196 \mathrm{~cm})$ was recorded in February at station A, while the lowest $(66 \mathrm{~cm})$ was recorded in June at station B. The highest total suspended solids value of $450 \mathrm{mgL}^{-1}$ was recorded in July at station A while the lowest value was in April at station B and $\mathrm{C}$ respectively. The total dissolved solids content was higher in the dry months than in the wet months. The valued were between $394 \mathrm{mgL}^{-1}$ in July at station $\mathrm{C}$ and $12546 \mathrm{mgL}^{-1}$ in March at station B. The $\mathrm{pH}$ values range between 6.23 in May at station $\mathrm{C}$ and 6.96 in May at station B. Conductivity values were higher in the dry months than in the wet months and ranged between $7.9 \times 10^{-4}$ and $2.5 \times 10^{-2} \mathrm{Scm}^{-1}$ during the study period. Dissolved oxygen values were between 3.2 in May at station B and $5.7 \mathrm{mgL}^{-1}$ in June at station C. Dissolved oxygen values were lower at station $\mathrm{A}$ than other stations. Salinity values ranged between $1.1 \%$ in July at station $\mathrm{A}$ and $\mathrm{C}$ and $12.1 \%$ in March at station B. Nitrate-nitrogen values were between $0.12 \mathrm{mgL}^{-1}$ in July at station $\mathrm{C}$ and $3.98 \mathrm{mgL}^{-1}$ in March at station $\mathrm{B}$. The 
highest phosphate-phosphorus value $\left(0.64 \mathrm{mgL}^{-1}\right)$ was recorded in March at station $\mathrm{B}$, with the lowest $\left(0.019 \mathrm{mgL}^{-1}\right)$ in July at station C. Sulphate values were higher in the dry months than in the wet months and ranged between $35.5 \mathrm{mgL}^{-1}$ in July at station $\mathrm{C}$ and $1135 \mathrm{mgL}^{-1}$ in March at station B. Heavy metal levels were high throughout the study, and higher levels were recorded in the dry than wet months. The highest Lead content $\left(0.78 \mathrm{mgL}^{-1}\right)$ was obtained in February at station $\mathrm{B}$, while the lowest value $\left(0.011 \mathrm{mgL}^{-1}\right)$ occurred in May at station B. The highest copper value recorded $\left(3.618 \mathrm{mgL}^{-1}\right)$ occurred in March at station A, while lowest value $\left(0.006 \mathrm{mgL}^{-1}\right)$ was in May at station $\mathrm{B}$. Iron values ranged between $0.06 \mathrm{mgL}^{-1}$ in June at station B and $3.618 \mathrm{mgL}$ in March at station A, respectively. The values for oil and grease were higher in the dry months than in the wet months. A range of between $9.0 \mathrm{mgL}^{-1}$ (occuring in March at station B) and $0.1 \mathrm{mgL}^{-1}$ (in July at station C) was recorded.

Table 1. Physico-chemical parameters in the Lagos Lagoon at Egbin (February to July, 2000).

\begin{tabular}{|c|c|c|c|c|c|c|c|c|c|c|c|c|c|c|c|c|c|c|}
\hline & \multicolumn{3}{|c|}{ February } & \multicolumn{3}{|c|}{ March } & \multicolumn{3}{|l|}{ April } & \multicolumn{3}{|l|}{ May } & \multicolumn{3}{|l|}{ June } & \multicolumn{3}{|l|}{ July } \\
\hline & A & B & $\mathrm{C}$ & $\mathrm{A}$ & B & $\mathrm{C}$ & A & B & C & A & B & C & A & $B$ & C & A & B & C \\
\hline Water temperature $\left({ }^{\circ} \mathrm{C}\right)$ & 36 & 32 & 27 & 37 & 34 & 29 & 38 & 36 & 29 & 40 & 38 & 30 & 38 & 34 & 27 & 35 & 34 & 27 \\
\hline Transparency $(\mathrm{cm})$ & 196 & 189 & 184 & 190 & 190 & 184 & 167 & 145 & 148 & 186 & 184 & 168 & 85 & 66 & 78 & 91 & 89 & 83 \\
\hline $\mathrm{pH}$ & 6.57 & 6.53 & 6.45 & 6.74 & 6.62 & 6.69 & 6.5 & 6.4 & 6.6 & 6.23 & 6.96 & 6.93 & 6.32 & 6.8 & 6.83 & 6.93 & 6.81 & 6 \\
\hline Total suspended Solids $\left(\mathrm{mgL}^{-1}\right)$ & 2 & 16 & 20 & 6 & 28 & 26 & 32 & 0 & 0 & 0 & 276 & 90 & 82 & 106 & 106 & 450 & 390 & 33 \\
\hline Total Dissolved Solids $\left(\mathrm{mgL}^{-1}\right)$ & 6496 & 7974 & 8082 & 11130 & 12546 & 1964 & 10036 & 9228 & 8960 & 9416 & 9080 & 8060 & 564 & 1198 & 798 & 402 & 410 & 39 \\
\hline Conductivity $\left(\mathrm{Scm}^{-1}\right)$ & 0.01 & 0.014 & 0.02 & 0.022 & 0.025 & 0 & 0.02 & 0 & 0.02 & 0.02 & 0.02 & 0.02 & 0.01 & 0.002 & 0 & 0 & $8 \mathrm{E}-04$ & 0 \\
\hline Salinity (\%o) & 6.5 & 6.7 & 7.7 & 10.1 & 12.1 & 7 & 9.3 & 7 & 7.4 & 7 & 6.7 & 7 & 1.4 & 1.4 & 1.4 & 1.1 & \begin{tabular}{|l|l|}
1.2 \\
\end{tabular} & 1 \\
\hline Dissolved Oxygen $\left(\mathrm{mgL}^{-1}\right)$ & 3.6 & 4.1 & 4.7 & 3.7 & 5.1 & 5.4 & 4.7 & 4.9 & 5.5 & 3.2 & 4.7 & 5.3 & 4.8 & 5.3 & 5.7 & 5 & 5.1 & 5 \\
\hline Phosphate $\left(\mathrm{mgL}^{-1}\right)$ & 0.33 & 0.36 & 0.42 & 0.57 & 0.64 & 0.12 & 0.528 & 0.52 & 0.48 & 0.48 & 0.47 & 0.41 & 0.04 & 0.067 & 0.04 & 0.03 & 0.022 & 0 \\
\hline Nitrate $\left(\mathrm{mgL}^{-1}\right)$ & 2.1 & 2.94 & 3.25 & 3.92 & 3.98 & 0.86 & 3.16 & 3.58 & 3.28 & 2.99 & 2.88 & 2.57 & 0.19 & 0.38 & 0.28 & 0.15 & 0.14 & 0 \\
\hline Sulphate $\left(\mathrm{mgL}^{-1}\right)$ & 628 & 715 & 745 & 1008 & 1135 & 179 & 922 & 184 & 817 & 852 & 821 & 735 & 51 & 108 & 72 & 38 & 37 & 35 \\
\hline Oil and grease $\left(\mathrm{mgL}^{-1}\right)$ & 6 & 6 & 7 & 8 & 9 & 4 & 4 & 6 & 6 & $\mathrm{ND}$ & $\mathrm{ND}$ & $\mathrm{ND}$ & 6 & 2 & 4 & 2 & 5 & 1 \\
\hline $\operatorname{Lead}\left(\mathrm{mgL}^{-1}\right)$ & 0.87 & 0.978 & 0.7 & 0.455 & 0.438 & 0.84 & 0.164 & 0.18 & 0.47 & 0.18 & 0.11 & 0.01 & 0.16 & 0.513 & 0.3 & 0.49 & 0.618 & 0 \\
\hline Iron $\left(\mathrm{mgL}^{-1}\right)$ & 7.44 & 7.866 & 8.64 & 12.62 & 10.67 & 6.11 & 6.646 & 5.76 & 0.92 & 4.62 & 0.1 & 0.62 & 2.69 & 2.316 & 0.78 & 8.64 & 16.48 & 10 \\
\hline Copper $\left(\mathrm{mgL}^{-1}\right)$ & 1.56 & 1.967 & 1.69 & 3.618 & 2.768 & 2.77 & 0.926 & 0.91 & 0.89 & 0.63 & 0.06 & 0.67 & 0.88 & 0.716 & 0.97 & 0.91 & 0.996 & 0 \\
\hline
\end{tabular}

\subsection{Sediment Characteristics}

Table 2 shows the Total organic matter in sediments (\%) at the study area. Total organic matter ranged between 0.1 and $0.3 \%$ which translate $402-6 \%$ dry weight. The percentage sediment texture composition, where as sand content varied from $49-97 \%$, clay ranged 0 and $28 \%$ and silt between 0 and $21 \%$. Total hydrocarbon content range between 0.14 and $0.32 \mathrm{mgl}^{-1}$ for station $\mathrm{A}, 0.03$ and $0.21 \mathrm{mgl}^{-1}$ for station B and 0.1 and $0.43 \mathrm{mgl}^{-1}$ for station C throughout the study (Table 3). Station A was predominantly sandy (78-97\%), clay (1 $13 \%)$, and silt $(3-21 \%)$. Station B was mixture of and clay, sand (56-79\%), clay (21 $33 \%)$ and silt $(0-19 \%)$. Station $C$ however had more of clay $(49-90 \%)$, sand $(4-36 \%)$ and silt $(1-15 \%)$. 
Table 2. Total Organic Matter in Sediments (\%) at Egbin, Lagos.

\begin{tabular}{|l|l|l|l|l|l|l|l|l|l|l|l|l|l|l|l|l|l|l|}
\hline Parameter & \multicolumn{4}{l}{ February } & \multicolumn{4}{l}{ March } & \multicolumn{4}{l|}{ April } & \multicolumn{3}{l|}{ May } & \multicolumn{3}{l|}{ June } \\
\hline Stations & A & B & C & A & B & C & A & B & C & A & B & C & A & B & C & A & B & C \\
\hline $\begin{array}{l}\text { Wt. } \\
\text { before } \\
\text { ignition } \\
\text { (g) }\end{array}$ & 5 & 5 & 5 & 5 & 5 & 5 & 5 & 5 & 5 & 5 & 5 & 5 & 5 & 5 & 5 & 5 & 5 & 5 \\
\hline $\begin{array}{l}\text { Wt. after } \\
\text { ignition } \\
\text { (g) }\end{array}$ & 4.9 & 4.9 & 4.9 & 4.8 & 4.8 & 4.9 & 4.9 & 4.9 & 4.7 & 4.9 & 4.9 & 4.7 & 4.9 & 4.7 & 4.7 & 4.7 & 4.8 & 4.8 \\
\hline Difference & 0.1 & 0.1 & 0.1 & 0.2 & 0.2 & 0.1 & 0.1 & 0.1 & 0.3 & 0.1 & 0.1 & 0.3 & 0.1 & 0.1 & 0.3 & 0.3 & 0.2 & 0.2 \\
\hline$\%$ & 2 & 2 & 2 & 4 & 4 & 2 & 2 & 2 & 6 & 2 & 2 & 6 & 2 & 2 & 6 & 6 & 4 & 4 \\
\hline
\end{tabular}

Table 3. Percentage Sediments texture at Egbin, Lagos.

\begin{tabular}{|c|c|c|c|c|c|c|c|c|c|c|c|c|c|c|c|c|c|c|}
\hline & \multicolumn{3}{|c|}{ February } & \multicolumn{3}{|c|}{ March } & \multicolumn{3}{|c|}{ April } & \multicolumn{3}{|c|}{ May } & \multicolumn{3}{|c|}{ June } & \multicolumn{3}{|c|}{ July } \\
\hline & A & B & $\mathrm{C}$ & A & B & $\mathrm{C}$ & $\mathrm{A}$ & $\mathrm{B}$ & $\mathrm{C}$ & A & B & $\mathrm{C}$ & A & $\mathrm{B}$ & $\mathrm{C}$ & $\mathrm{A}$ & B & $\mathrm{C}$ \\
\hline Sand & 83 & 79 & 94 & 94 & 74 & 75 & 96 & 68 & 68 & 71 & 78 & 65 & 95 & 83 & 49 & 97 & 66 & 83 \\
\hline Clay & 12 & 21 & 0 & 0 & 23 & 24 & 0 & 25 & 29 & 1 & 15 & 28 & 5 & 13 & 36 & 0 & 15 & 13 \\
\hline Silt & 4 & 0 & 6 & 6 & 63 & 1 & 4 & 7 & 3 & 21 & 7 & 7 & 0 & 4 & 15 & 3 & 19 & 4 \\
\hline
\end{tabular}

\subsection{Biological Characteristics}

\subsubsection{Phytoplankton Spectrum}

A total of 83 phytoplankton species from 43 genera were recorded in this study (Table 4). The diatoms were represented by 50 species from 22 genera while the cyanobacteria comprised by 24 species from 13 genera, and the green algae made up seven species from six genera. The euglenoids were represented by two species from a genus. For the diatoms the pinnate forms (32 species) were more abundant than the centric forms (18 species). Aulacoseira grannulata and A. granulate var. augustissima were notable species in the wet month. Other frequent species were Melosiira moniliformis, Coscinodiscus centralis, Coscinodiscus eccentricus, Biddulphia aurita, Terpsinoe musica, Thalassionema fraunfeldii, Gyrosigma balticum, Pinnularia major, Pleurosigma angulatum, Synedra crystalline, Pinnularia major and Pleurosigma angulatum. For the cyanobacteria the heterocytous forms (15 species) were more frequent than the chrococcoid forms (9 species). Station A recorded higher diversity than station B and C. Cyanobacteria species comprised Pseudanabaena galeata, Microcystis flos-aquae, Lyngbya martensiana, Aphanocapsa sp., Calothrix coenfervicola, Phormidium uncinatum, Schizothrix fasciculata, Oscillatoria formosa and Oscillatoria minima.The green algae $(7$ species) were only recorded in the wet months. Gonatozygon and Spirogyra sp. were the abundant green algae species recorded. Other frequent species included Cladophora glomerata, Cladophora sp., Oedogonium sp. and Rhizoclonium sp. 
Table 4. Phytoplankton of a waste heat discharge area at Egbin, Lagos

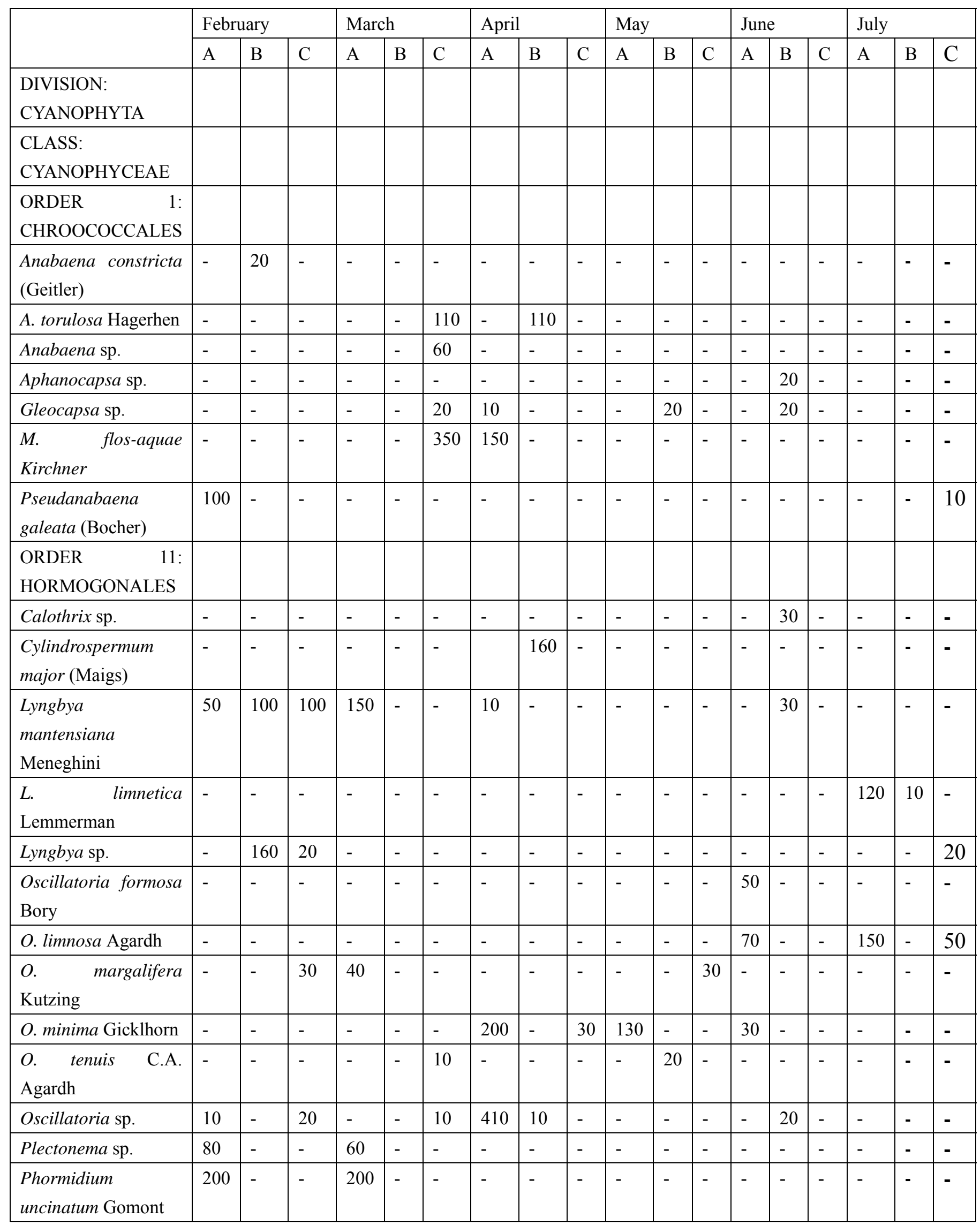




\section{Macrothink}

\begin{tabular}{|l|l|l|l|l|l|l|l|l|l|l|l|l|l|l|l|l|l|l|}
\hline $\begin{array}{l}\text { Schizothrix } \\
\text { fasciculate Gomont }\end{array}$ & - & - & - & - & - & - & - & - & - & 30 & - & - & - & - & - & - & - & - \\
\hline Tolypothrix sp. & 40 & - & - & - & - & - & - & - & - & - & - & - & - & - & - & - & - & - \\
\hline $\begin{array}{l}\text { CLASS: } \\
\text { CHLOROPHYCEAE }\end{array}$ & & & & & & & & & & & & & & & & & & \\
\hline $\begin{array}{l}\text { Cladophora } \\
\text { glomerata (L) } \\
\text { Kutzing }\end{array}$ & - & - & 40 & - & 90 & - & 130 & - & - & - & 30 & - & - & - & 60 & - & - & - \\
\hline Cladophora sp. & - & - & 30 & - & - & - & 70 & - & - & - & - & - & - & 70 & 10 & - & - & - \\
\hline Rhizoclonium sp. & - & - & 50 & - & - & - & - & - & - & - & - & - & - & - & - & - & - & - \\
\hline
\end{tabular}

\subsubsection{Phytoplankton Community Structure}

The phytoplankton community structure is presented in Table 5. Shannon and weaver index (HS) was low $(<1.0)$ all through the study except in February and May values of 1.06 and 1.12 recorded in February at stations $\mathrm{A}$ and $\mathrm{C}$ and 1.02 recorded in May at station $\mathrm{C}$. Similarly, the simple diversity index $(\mathrm{d})$ was low $(\leq 0.60)$ all through the study period.

Table 5. Phytoplankton Community Structure at waste heat discharge site at Egbin, Lagos

\begin{tabular}{|c|c|c|c|c|c|c|c|c|c|c|c|c|c|c|c|c|c|c|}
\hline & \multicolumn{3}{|c|}{ February } & \multicolumn{3}{|c|}{ March } & \multicolumn{3}{|l|}{ April } & \multicolumn{3}{|l|}{ May } & \multicolumn{3}{|l|}{ June } & \multicolumn{3}{|l|}{ July } \\
\hline & A & B & $\mathrm{C}$ & A & B & $\mathrm{C}$ & A & B & $\mathrm{C}$ & A & B & $\mathrm{C}$ & A & B & $\mathrm{C}$ & A & B & $\mathrm{C}$ \\
\hline $\begin{array}{l}\text { Total } \\
\text { Phytoplankton } \\
\text { diversity (S) }\end{array}$ & 17 & 12 & 16 & 7 & 11 & 8 & 10 & 13 & 7 & 14 & 8 & 14 & 8 & 13 & 7 & 10 & 9 & 17 \\
\hline $\begin{array}{l}\text { Total } \\
\text { Phytoplankton } \\
\text { abundance } \\
\text { (N) }\end{array}$ & 1460 & 870 & 540 & 560 & 890 & 600 & 1080 & 540 & 240 & 470 & 180 & 910 & 4410 & 1150 & 41520 & 43620 & 370 & 35080 \\
\hline $\begin{array}{l}\text { Log of } \\
\text { Species } \\
\text { diversity }\end{array}$ & 1.23 & 1.08 & 1.20 & 0.85 & 1.04 & 0.90 & 1.00 & 1.11 & 0.85 & 1.15 & 0.90 & 1.15 & 0.90 & 1.11 & 0.85 & 1.00 & 0.95 & 1.23 \\
\hline $\begin{array}{l}\text { Log of } \\
\text { phytoplankton } \\
\text { abundance }\end{array}$ & 3.16 & 2.94 & 2.73 & 2.55 & 2.95 & 2.78 & 3.03 & 2.73 & 2.38 & 2.67 & 2.26 & 2.96 & 3.64 & 3.06 & 4.62 & 4.64 & 2.57 & 4.55 \\
\hline $\begin{array}{l}\text { Shannon and } \\
\text { Weaver Index } \\
\text { (Hs) }\end{array}$ & 10.6 & 0.88 & 1.12 & 0.72 & 0.82 & 0.57 & 0.77 & 0.92 & 0.67 & 0.98 & 0.82 & 1.02 & 0.64 & 0.93 & 0.50 & 0.49 & 0.74 & 0.47 \\
\hline $\begin{array}{l}\text { Margalef } \\
\text { index }\end{array}$ & 2.20 & 1.63 & 2.38 & 0.95 & 1.47 & 1.09 & 1.29 & 1.91 & 1.09 & 2.12 & 1.35 & 1.91 & 0.83 & 1.70 & 0.56 & 0.84 & 1.35 & 1.53 \\
\hline $\begin{array}{l}\text { Equitability } \\
\text { Index (j) }\end{array}$ & 0.886 & 0.81 & 0.93 & 0.85 & 0.78 & 0.64 & 0.77 & 0.82 & 0.80 & 0.86 & 0.90 & 0.89 & 0.71 & 0.83 & 0.59 & 0.49 & 0.24 & 0.42 \\
\hline
\end{tabular}




\subsubsection{Zooplankton Composition}

Zooplankton composition is presented on Table 6. Species identified were Diphanosona sp., Chdorus sp., and Bosmina sp. The amphipods were represented by Grammarus sp., Hyperia galba, the Mysids comprised Mysis oculata, the Chaetognaths were represented by Sagitta enflata while the Cnidarians consisted of Chsaora melananster and Sarsia eximia similar observations have been repointed by Sudhankar (2010). The zooplankton community structure is presented in Table 7. These indices values showed both spatial and temporal variations.

Table 6. Zooplankton of a waste heat discharge area at Egbin, Lagos

\begin{tabular}{|l|}
\hline Phylum: Arthropoda \\
\hline Class I: Crustacea \\
\hline Order I: Calanoida \\
Acartia clausii Giesbrecht \\
Paracalanus parvus (Claus) \\
Centropage typicus Dana \\
Diaptomus sp. \\
Eurtemora sp. \\
Isias claripes Boeck \\
Microcalamus sp. \\
Pseudocalanus elongates Boeck \\
Temora longicornis M. H. Doall \\
\hline \\
\hline Order II: Cyclopoida \\
Cyclops sp. \\
Oithona nana (Grisbrecht) \\
\hline \\
\hline Order III: Cladocera \\
Bosmina sp. \\
Chdorus sp. \\
Daphnia sp. \\
Diaphanosoma sp. \\
\hline \\
\hline Order IV: Amphipoda \\
Gammarus sp. \\
Hyperia galba (Mont.) \\
\hline \\
\hline Order V: Paracarida \\
Sub-order: Mysidacea \\
Mysis oculata (Fabricius) \\
\hline \\
\hline Phylum: Chaetognatha \\
Sagitta enflata Vogt \\
\hline Class II: Hydrozoa \\
Order: Siphonophora \\
Chysaora melanaster Brandt \\
Sarsia eximia (Atlman) \\
\hline
\end{tabular}




\section{Ml Macrothink}

The zooplankton community structure is presented in Table 7. Margalef index (d) was high $(>1.0)$ in February and March at station A and at station A and B in June and July. Shannon and Weaves index (HS) was low $(<4.0)$ at station B in between March and May. HS was also low at all station in June and station C in July. Except in May at station B, equitability was high $(>0.80)$ all through the sampling period.

Table 7. Zooplankton Community Structure around a waste heat discharge area at Egbin, Lagos

\begin{tabular}{|c|c|c|c|c|c|c|c|c|c|c|c|c|c|c|c|c|c|c|}
\hline & \multicolumn{3}{|c|}{ February } & \multicolumn{3}{|c|}{ March } & \multicolumn{3}{|l|}{ April } & \multicolumn{3}{|l|}{ May } & \multicolumn{3}{|l|}{ June } & \multicolumn{3}{|l|}{ July } \\
\hline & A & B & $\mathrm{C}$ & A & B & $\mathrm{C}$ & A & B & $\mathrm{C}$ & A & B & $\mathrm{C}$ & A & B & C & A & B & $\mathrm{C}$ \\
\hline $\begin{array}{l}\text { Total } \\
\text { zooplankton } \\
\text { diversity } \\
\text { (S) }\end{array}$ & 8 & 5 & 5 & 7 & 4 & 5 & 5 & 3 & 5 & 3 & 1 & 3 & 8 & 6 & 3 & 5 & 6 & 3 \\
\hline $\begin{array}{l}\text { Total } \\
\text { zooplankton } \\
\text { abundance } \\
\text { (N) }\end{array}$ & 280 & 260 & 190 & 340 & 100 & 100 & 70 & 50 & 90 & 50 & 10 & 30 & 180 & 150 & 50 & 90 & 130 & 30 \\
\hline $\begin{array}{l}\text { Log of } \\
\text { Species } \\
\text { diversity }\end{array}$ & 0.90 & 0.70 & 0.70 & 0.85 & 0.60 & 0.70 & 0.70 & 0.48 & 0.70 & 0.48 & 0.00 & 0.48 & 0.90 & 0.78 & 0.48 & 0.70 & 0.78 & 0.48 \\
\hline $\begin{array}{l}\text { Log of } \\
\text { zooplankton } \\
\text { abundance }\end{array}$ & 2.45 & 2.41 & 2.28 & 2.53 & 2.00 & 2.00 & 1.85 & 1.70 & 1.95 & 1.70 & 1.00 & 1.48 & 2.26 & 2.18 & 1.70 & 1.95 & 2.11 & 1.48 \\
\hline $\begin{array}{l}\text { Shannon } \\
\text { and Weaver } \\
\text { Index (Hs) }\end{array}$ & 0.64 & 0.39 & 0.59 & 0.60 & 0.52 & 0.65 & 0.64 & 0.46 & 0.62 & 0.41 & 0.00 & 0.48 & 0.84 & 0.70 & 0.46 & 0.64 & 0.74 & 0.48 \\
\hline $\begin{array}{l}\text { Margalef } \\
\text { index }\end{array}$ & 1.24 & 0.72 & 0.76 & 1.03 & 0.65 & 0.89 & 0.94 & 0.51 & 0.89 & 0.51 & 0.00 & 0.59 & 1.35 & 1.00 & 0.51 & 0.89 & 1.03 & 0.59 \\
\hline $\begin{array}{l}\text { Equitability } \\
\text { Index (j) }\end{array}$ & 0.71 & 0.55 & 0.84 & 0.70 & 0.86 & 0.93 & 0.92 & 0.96 & 0.89 & 0.86 & 0.00 & 1.00 & 0.93 & 0.90 & 0.96 & 0.91 & 0.95 & 1.00 \\
\hline
\end{tabular}

\subsubsection{Benthic}

A total of five macro-fauna characteristics were recorded (Table 8). All species belonged to the Phylum mollusca. Whereas the Gastropods made up three species (Pachymelania aurita, Tympanotonus fuscaatus and Neritina glabrata), the bivalves accounted for two species (Iphigenia truncate and Aloidis trigona). The Pachymelania aurita and Tympanotonus fuscantus var. radula were the more abundant and more frequent species, followed by Aloidis trigona. The benthic fauna community structure is presented in Table 9. These indices showed both spatial and temporal variations. 
Table 8. Benthic Macrofauna around a waste heat discharge area at Egbin, Lagos

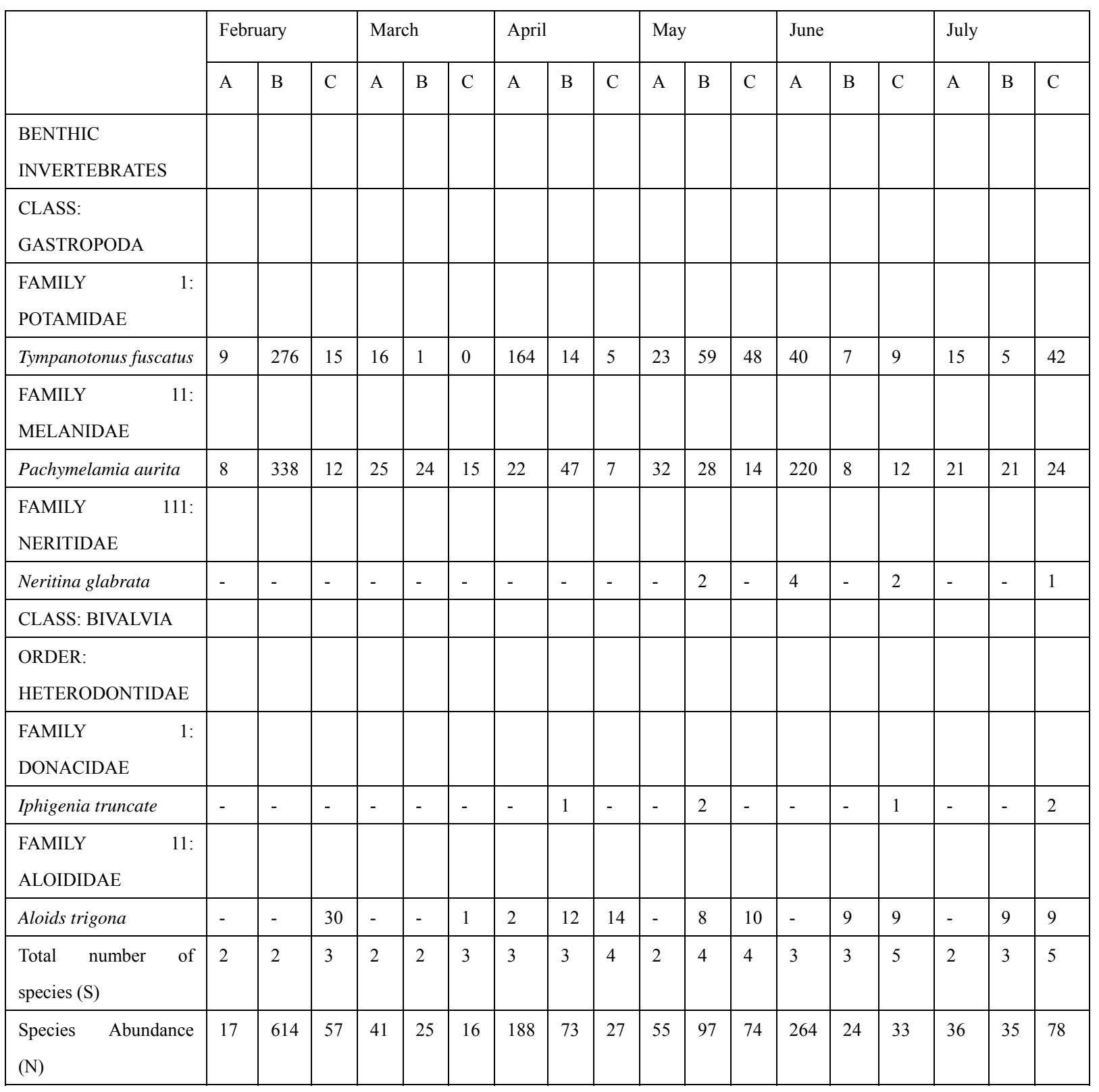


Table 9. Macrofauna community structure of a waste head discharge area at Egbin, Lagos

\begin{tabular}{|c|c|c|c|c|c|c|c|c|c|c|c|c|c|c|c|c|c|c|}
\hline & \multicolumn{3}{|c|}{ February } & \multicolumn{3}{|c|}{ March } & \multicolumn{3}{|c|}{ April } & \multicolumn{3}{|l|}{ May } & \multicolumn{3}{|l|}{ June } & \multicolumn{3}{|l|}{ July } \\
\hline & A & B & $\mathrm{C}$ & A & B & C & A & B & $\mathrm{C}$ & A & B & $\mathrm{C}$ & A & B & $\mathrm{C}$ & A & B & $\mathrm{C}$ \\
\hline $\begin{array}{l}\text { Total species } \\
\text { diversity }(\mathrm{S})\end{array}$ & 2 & 2 & 3 & 2 & 2 & 3 & 3 & 3 & 4 & 2 & 4 & & 4 & 3 & 5 & 2 & 3 & 5 \\
\hline $\begin{array}{l}\text { Total abundance } \\
\text { (N) }\end{array}$ & 17 & 614 & 57 & 41 & 25 & 16 & 188 & 73 & 27 & 55 & 97 & 74 & 264 & 24 & 33 & 36 & 35 & 78 \\
\hline $\begin{array}{l}\text { Log of Species } \\
\text { diversity }\end{array}$ & 0.30 & 0.30 & 0.48 & 0.30 & 0.30 & 0.48 & 0.48 & 0.48 & 0.60 & 0.30 & 0.60 & 0.60 & 0.48 & 0.48 & 0.70 & 0.30 & 0.48 & 0.70 \\
\hline $\begin{array}{ll}\log & \text { of } \\
\text { abundance } & \end{array}$ & 1.23 & 2.79 & 1.76 & 1.61 & 1.40 & 1.20 & 2.27 & 1.89 & 1.43 & 1.74 & 1.99 & 1.87 & 2.42 & 1.38 & 1.52 & 1.56 & 1.54 & 1.89 \\
\hline $\begin{array}{l}\text { Shannon-Weiner } \\
\text { index }(\mathrm{Hs})\end{array}$ & 0.30 & 0.30 & 0.44 & 0.29 & 0.07 & 0.10 & 0.18 & 0.39 & 0.49 & 0.30 & 0.41 & 0.42 & 0.22 & 0.47 & 0.59 & 0.29 & 0.41 & 0.48 \\
\hline $\begin{array}{l}\text { Margalef index } \\
\text { (d) }\end{array}$ & 0.35 & 0.16 & 0.49 & 0.27 & 0.31 & 0.72 & 0.38 & 0.47 & 0.91 & 0.25 & 0.26 & 0.70 & 0.36 & 0.63 & 1.14 & 0.28 & 0.56 & 0.92 \\
\hline $\begin{array}{l}\text { Equitability } \\
\text { Index (j) }\end{array}$ & 1.00 & 0.99 & 0.93 & 0.96 & 0.24 & 0.21 & 0.38 & 0.82 & 0.81 & 0.98 & 0.96 & 0.70 & 0.46 & 1.00 & 0.84 & 0.98 & 0.85 & 0.86 \\
\hline $\begin{array}{l}\text { Simpson's } \\
\text { dominance } \\
\text { Index (C) }\end{array}$ & 0.50 & 0.51 & 0.11 & 0.52 & 0.92 & 0.88 & 0.77 & 0.45 & 0.10 & 0.51 & 0.45 & 0.46 & 0.72 & 0.20 & 0.21 & 0.51 & 0.38 & 0.39 \\
\hline
\end{tabular}

\section{Discussion}

Higher temperature values recorded at the Egbin part of Lagos lagoon may be attributed to the effect of waste heat discharged from the thermal facility into the lagoon. The temperature ranged between $27-40^{\circ} \mathrm{C}$ possibly reflecting a reduction as one moved further away from the thermal plants. Observed variation in other physical and chemical characteristics of the lagoon at station C reflected similar trends for the lagoon at Lagos and Epe (Nwankwo 1996, 1998, Onyema et al., 2003). The effect of temperature on water and benthic organisms has been documented by some authors Roessler and Durbin, 1974, Pandey, 1983). There was low dissolved oxygen levels particularly at station A that had the highest temperature readings. Increased temperature may have increased the metabolic rate of associated organisms that could have induced higher utilization of oxygen. The highest transparency recorded in station A could be due to suspended matter going into solution as a result of increased temperatures $\left(40^{\circ} \mathrm{C}\right)$. According to Thomas (1966), higher temperatures of the dry season increased oxidation of organic matter in a man-made lake in Ghana. However, the higher transparencies at the three stations in the dry months may also be due to cessation of flood water input and the incursion sea water. High transparencies in the dry season have been reported in the Lagos lagoon (Olaniyan 1969, Oyenekan 1975, Nwankwo 1996, 1998, Chukwu and Nwankwo 2004). According to Ajao et al., (1996), in addition to waste heat, the Lagos lagoon at Egbin experiences such contaminants as metals, oil and grease. In this study high heavy metal levels were reported especially at station $\mathrm{A}$ in the dry months. The $\mathrm{pH}$ was acidic ranging between 6.23 and 6.96 the high metal concentration at station A could be a 
combination of fallout from the facility and acid waste induced leaching from sediments. Acid water induce leaching from sediments had been reputed by (Oyenekan 1987). Salinity was high throughout the study at all stations, except in June and July where it fell probably due to the dilution effect of rainfall induced flood waters. June and July fell within the first annual rainfall peak in Southwest Nigeria (Nwankwo 1986). The high salinity values recorded in the dry months could in addition be due to effluent from the facility. Thermal plants are known to discharge high saline waters as well as to heat (Odiete, 1999). Rainfall in coastal waters of South west Nigerian dilutes water characteristics, breaks down horizontal radients and flushes fresh water biota into the sea (Nwankwo 1996, 1998). The reduction in nitrate-nitrogen, phosphate-phosphorus, sulphate values and the increased total suspended solids values observed in June and July may be attributed to dilution from floodwater and the introduction of allochtonous material from adjoining wetlands. According to Dance (1981), the transportation of particulate matter in streams is a physical process while the transportation of particulate organic matter is positively related to precipitation and flow conditions. According to Nwankwo (1998) Aulacoseira granulata (Ehr.) Ralf and A. granulata var. angustissima are prominent taxa in the eastern zone of the Lagos lagoon system which is primarily fresh or low brackish. Similar floral spectrum was observed in this study with Gonatozygon sp as a sub-dominant species. These taxa could be indicators of fresh water/acidic to neutral conditions. Brackish water indicators observed were Biddulphia aurila, B. laevis, Gyrosigina balticum, Melosira moniliformis, M. nummuloides while cyanobacteria bloom species recorded included Microcystis aeruginosa and M. flos-aquae (Nwankwo 1993, 1996, 1996), Nwankwo (1993), suggested that heavy metals levels have increased greatly in the Lagos lagoon possibly due to formation of forming complexes that could make some compounds unavailable to the phytoplankton. High copper values $\left(\geq 1.0 \mathrm{mgL}^{-1}\right)$, above acceptable FEPA (1991) limits for coastal waters were recorded at the Egbin part of the Lagos lagoon. In this study, the composition and abundance of cyanobacteria were positively correlated with water temperature $(0.68)$ at station A. Cyanobacteria are opportunistic species that exploit environments with elevated temperatures (Van Landingham 1962). Similarly, Kadiri (1999) reported high cyanobacteria diversity in a warm spring at Ikogosi, Nigeria. The observed dominance of diatoms and copepods confirm reports for the Lagos lagoon (Nwankwo 1996, 1998, Onyema et al., 2003). In the Lagos lagoon, the organic matter distribution is influenced by anthropogenic input and deposition pattern which in turn are regulated by tidal dynamics (Ajao et al., 1996). Total organic matter in the stations was low. The relatively higher TOM content $(60 \%)$ recorded at station $\mathrm{C}$ may not be enough to support the views of Bader et al., (1970) as cited by Oyenekan (1998) that TOM content could be an index quantity of food available to benthic fauna. Although the number of benthic organisms recorded at station $\mathrm{C}$ was higher than at station $\mathrm{A}$ and $\mathrm{B}$, they do not compare to higher values for other sources of point pollution in the Lagos lagoon (Nwankwo 1986, Nwankwo 1998, Nwankwo et al., 2008, Onyema et al., 2003). According to Ajao and Fagade (1990), Brown and Oyenekan (1988), benthic organisms in the Lagos lagoon are sediment type specific. The benthic macro fauna in this study were dominated by the gastropod Pachymelania aurila. Gray (1979) correlated changes in community structure with environmental perturbations. Decline in diversity could be linked to thermal stress and commonly practiced sand extraction 
that altered the sediment type. The numerous empty shells recorded at all stations in the study may be a consequence of waste heat induced elevated temperatures and possibly mimicking the effect of global warming. The benthic community at Egbin was similar to the Pachymelania community described by Oyenekan (1988) as being the dominant macro fauna community in the Lagos lagoon. In this study, the low species diversity recorded in the plankton and benthic fauna, may be a consequence of the effect of elevated water temperatures that may have led to high mortality and empty shell numbers, low dissolved oxygen, reduced organic content and acidic conditions.

\section{Acknowledgements}

The authors wish to thank Dr (Mrs) Adesalu T.A of department of Botany for her support, the Department of Marine Sciences for providing equipments and Late Mr Emubaiye for logistic support.

\section{References}

Abbaspour, M., Javid A.H., Mohinmi P., \& Kayham K. (2005). Modeling of thermal pollution in coastal area and its economical and environmental assessment. International Journal of Environmental Science and Technology, 2, 13 - 26. http://dx.doi.org/10.1007/BF03325853

Ajao, E.O, Oyewo E., \& Uniyinadu (1996). A Review of the pollution of coastal waters in Nigeria. NIOMR Technical Paper No. 107, 20pp.

Akpata, T. V. I., Oyenekan, J. A., \& Nwankwo, D. I. (1993). Impact of organic pollution on the bacteria, plankton and benthic population of Lagos Lagoon, Nigeria. International Journal of Ecology and Environmental Sciences, 19, 73 - 82.

APHA (1998). Standard methods for the examination of water and waste water $20^{\text {th }}$ Edition. American Public and Health Association.

Bader, R. G., \& Roesler, M. A. (1971). Thermal pollution of a tropical Marine estuary marine pollution and sea life. Fishing News (books) Ltd., NewYork 70.

Brown, C. A., \& Oyenekan, J. A. (1998). Temporal variation in the structure of macrobenthic invertebrate communities of the Lagos lagoon; Nigeria. Polish Acta Hydrobiologia, 45, $45-57$.

Brown, C. A. (1998). Distribution and population dynamic of an estuaries population of Aloidis trigona Hinds (Bivalva) Acta Hydrobioogia, 40, 227-237.

Dancem K. W. (1981). Seasonal Aspect of transport of organic matter in streams. In: Prospective in Mining water Ecology. M.A. Lock (ed) Plenium. Press NewYork 61-95. http://dx.doi.org/10.1007/978-1-4684-1122-5_4

Edmunds, J. (1978). Sea shores and mollusks found on West African coasts and estuaries. Ghana University Press, Accra. 146.

Egborge, A. B. M. (1979). The effect of Impoundment on the phytoplankton of the River Oshun Nigeria. Nova Hedwigia, 31, 407- 418. 
Emmanuel, B. E., \& Onyema, I. C. (2007). The Plankton and fishes of a tropical creek in South western Nigeria. Tropical Journal of Fisheries and Aquatic Sciences, 7, 105-114.

Food and Agricultural Organisation. (1969). Fisheries survey in the West and Mid-western region of Nigeria FAO/SF 74 NIR. 6: 142pp.

Hendey, N. I. (1964). An Introductory Account of the small algae of the British coastal water. Part V. Bacillanophyceae (Diatoms) Ministry of Agriculture, Fisheries and Food London. Fisheries Investigations series, 4, 317.

Hill, M. B., \& Webb. (1958). The ecology of the Lagos Lagoon II. The topography and physical features of the Lagos harbor and Lagos Lagoon Philosophical transaction of Royal Society London. Series B, Biological Sciences, 241, 319 -333. http://dx.doi.org/10.1098/rstb.1958.0006

Huntley, M. E., \& Lopez, M. D. G. (1992). Temperature dependent production of Marine copepods: a global synthesis. Am. Natural, 140, 201-242. http://dx.doi.org/10.1086/285410

Kadiri, M. O. (1999). Phytoplankton distribution in some coastal waters of Nigeria Nigerian Journal of Botany, 12, $51-62$.

Kannan L. (2005). Heavy metal concentrations in Ariyankuppan estuary and Verampattinam coast of Pondicherry. Indian Journal of Fisheries, 52, 501-506.

Lackey, J. B. (1938). The manipulation and country of river plankton and changes in some organisms due to formalin preservation: United States Public Health reports, 63, 2086 - 2093.

Macereth, F. J. H. (1971). Water Analysis for Limnologists. Freshwater Biological Association. Windermere, England.

Margalef, R. (1951). Diversidae despecies en les communides natureales. Publ. Int. Biol. Barcelona, 9, 5-27.

Nwankwo, D. I. (1986). Phytoplankton of a sewage disposal site in Lagos Lagoon, Nigeria Algae Nig. J. Biol Sci. 1(2), 89 - 96

Nwankwo, D. I. (1993). Cyanobacteria bloom species in coastal waters on south western Nigeria. Arch. Hydrobiol. (Suppl. 90) 4, 533-541

Nwankwo, D. I. (1996). Phytoplankton Diversity and Succession in Lagos Lagoon, Nigeria. Arch. Hydrobiol, 135(4), 529-542.

Nwankwo, D. I. (1998). Seasonal changes in phytoplankton population and diversity in Epe Lagoon, Nigeria: Acta Hydrobiol, 10(2), 83-92.

Nwankwo, D.I. (1998). The influence of sawmill wood wastes on diatom populations at Oko-baba, Lagos, Nigeria. Nig. J. Bot. 11, 15-24.

Nwankwo, D. I. (2004). The microalgae: our in dispensable allies in Aquatic monitoring and Biodiversity sustainability. Inaugural Lecture series. University of Lagos Press. 44. 
Nwankwo, D. I., \& Adesalu, T.A. (2010). Notes on indicators of environmental states in inshore and offshore waters of south-western Nigeria. Nature and Science, 8(6), $62-65$.

Odiete, W. O. (1999). Environmental Physiology of Animals and Pollution Diversified. Resource Ltd, Lagos 261pp.

Olaniyan, C. I. O. (1969). Seasonal variations in the hydrology and total plankton of Lagoons of South-western Nigeria. Nig. J. Sci, 3(2), $101-129$

Olaniyan, C. I. O. (1975). An Introduction to West African Animal Ecology. Henneman Publishers, Ibadan. 167pp.

Onyema, I.C., Otudeko, O.G., \& Nwankwo, D.I. (2003). The distribution and composition of plankton around a sewage disposal site at Iddo. J. Sci. Res. Dev. 7, $11-24$.

Oyenekan, J.A. (1975). A study of the Lagos Lagoon benthos (with particular reference to the molluscan) M.SC Dissertation University of Lagos, Nigeria 137pp.

Oyenekan, J.A. (1987). Benthic macrofaunal community of Lagos Lagoon, Nig. J. Sci., 21, $45-51$.

Patrick, R., \& Reimer, C.W. (1966 - 1975). The diatoms of the United States 2 vols. Monograph of the Academy of Natural sciences. Philadelphia 13

Schoemann, L. J., Becquevort, V. S., Stefels, J., Rousseau, V., \& Lancelot, C. (2005). Phaeocystis blooms in the global ocean and their controlling mechanisms: a review. J. Sea Res., Spec., 53(1-2), 43-66. http://dx.doi.org/10.1016/j.seares.2004.01.008

Shannon, C. E., \& Weaver, E. (1963). The Mathematical Theory of communications. University of Ilinois Press. 11.

Straughan, D. (1980). Impact of Southern Califonia Edison's operations on Intertidal Harbour, Southwestern Califonia Development Series, 80 - RD-95

Suresh, K., Ahamed, M.S., Durairaj, G., \& Nair, K.V.K. (1993). Impact of power plant heated effluent on the abundance of sedimentary organisms, off Kalpakkam, East Coast India. Hydrobiol., 268, 109 - 114. http://dx.doi.org/10.1007/BF00006881

Swamy, G. N. (1994). Dispersion processes in coastal waters - some outstanding practical issues for monitoring and modelling. Training Programme in Modelling and Monitoring of Coastal Marine Pollution (MAMCOMP). National Institute of Oceanography, Goa, India. 21 Nov. - 16 Dec., Indian Inst. Of Technol., New Delhi NIO, India. Pp: 595 - 597.

Thomas, J. D. (1966). Some preliminary observations on the fauna and flora of a small man-made lake in the West Africa Savanah. Bull delFAO

Thorhaug, A. (1978). The effect of heated effluent from power plants on sea grass (Thalassia) communities quantitatively comparing estuaries in the subtropics to the tropics. Mar. Pollut. Bull., 9, 181 - 187. http://dx.doi.org/10.1016/0025-326X(78)90175-3

Ukuoma, O. (1989). Thermal Pollution Effect on water quality and aquatics life Paper 
presented at a conference on watest pollution at Port-Harcourt, Nigeria. $19^{\text {th }}$ October, 1989. 32pp.

Valandingham, S. L. (1982). Guide to the identification and environment required and pollution tolerance of fresh-water, Blue-green algae (cyanophyta) U.SA. protection Agency. EPA - 60 .

Waife, G., \& Frid, C. L. J. (2001). Marine zooplankton and west Africa Marine Biodiversity capacity building in the West African Sub-region. Darion Initiative Reports 12699. 